\title{
Erratum: Anharmonicity and Universal Response of Linear Carbon Chain Mechanical Properties under Hydrostatic Pressure [Phys. Rev. Lett. 125, 105501 (2020)]
}

Keshav Sharma, Nathalia L. Costa, Yoong Ahm Kim, Hiroyuki Muramatsu, Newton M. Barbosa Neto, Luiz G. P. Martins, Jing Kong, Alexandre Rocha Paschoal, and Paulo T. Araujo

(Received 3 January 2022; published 27 January 2022)

DOI: 10.1103/PhysRevLett.128.049901

The symbol $\%$ in the vertical axis' label $[\varepsilon(\%)]$ of Fig. 2(b) of this Letter is a typographical error and leads to a wrong evaluation of the strains obtained from the experiments. The correct label must read $\varepsilon$ and the range of values from 0.00 to -0.03 must mean, in percentage, the range of values from 0 to $-3 \%$.

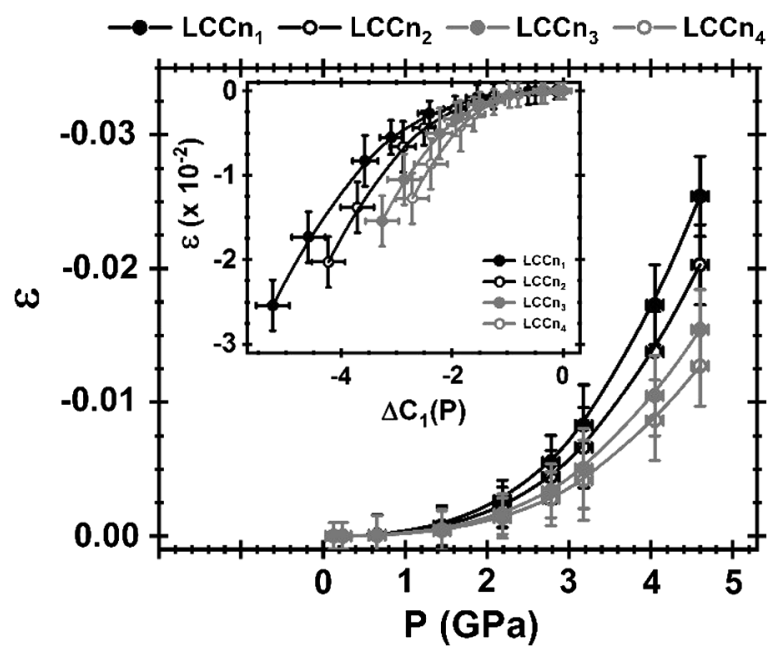

FIG. 2 (a) Young's modulus $E$, (b) strain $\varepsilon$, and (c) Grüneisen parameter $\gamma$ as a function of $P$ for each LCC. Both $E$ and $\gamma$ follow a $P^{-1}$ universal law, while $\varepsilon$ follows $P^{2}$ universal law. data (d) $(\Delta \omega / \omega)(E / \gamma)=-P$, an important parameter nanometrology, is universal and unified. (a)-(d) the solid lines are experimental data fittings. Insets: (a) shows the $E(P)$ vs $P$ graphic enlarged to highlight the $P \rightarrow \infty$ limit. The horizontal dashed lines correspond to the values $E$ (4.60 GPa) for each LLC. (b) shows the evolution of $\varepsilon$ with relation to the relative changes of $C_{1}$ with increasing $P$. (c) shows the $\gamma(P)$ vs $P$ graphic zoomed to highlight the $P \rightarrow \infty$ limit. The horizontal dashed lines correspond to the values of $\gamma$ calculated with $E=0.3$ TPa for each LCC [1]. The vertical dashed lines in (a) and (c) stand for $P_{c}=0.1 \mathrm{GPa}$.

[1] S. Kotrechko, I. Mikhailovskij, T. Mazilova, E. Sadanov, A. Timoshevskii, N. Stetsenko, and Y. Matviychuk, Mechanical properties of carbyne: Experiment and simulations, Nanoscale Res. Lett. 10, 24 (2015). 